\title{
THE THERAPEUTIC POSSIBILITIES OF SURGICAL BIO-ENGINEERING IN INCOMPLETE SPINAL CORD LESIONS
}

\author{
(Spinal Cord Relaxation in the Surgical Treatment of \\ INCOMPlete SPINAL CORD Lesions)
}

\author{
By Alf BREIG, M.D. \\ Sjukhuset, Stockholm, Sweden
}

THE aspect of bio-engineering I am going to describe can be described briefly as spinal cord relaxation.

In surgical operations on the skeletal system our approach is governed by well understood principles. In the case of the spinal cord, however, our knowledge of the effect of pathological changes on the nerve parenchyma, unfortunately, leaves much to be desired.

It is a generally accepted fact that mechanical stress on the ulnar nerve can be relieved by transposition and that here the underlying mechanism is relaxation of the nerve tissue. On the other hand, no one appears to have considered the possibility that the detrimental forces acting on the soft parenchyma of the spinal cord can also be eliminated by corrective surgery. It is true that in surgery today there is a tendency to intervene and correct anomalous functional processes in soft tissue organs, for instance, heart valve surgery and in various shunt operations for hydrocephalus.

To visualise the kinds of pathological stress in the cord tissue that occurs in practice I have recently reproduced as accurately as possible in situ in the cadaver the forces to which the cord is subjected, when the vertebral column is $(a)$ dorsiflexed and $(b)$ ventriflexed. While subjected to stress, the cord, whose blood vessels had previously been filled with radio-opaque medium, was fixed with formalin. In this way it was possible to conduct a thorough examination of the deformation of the cord tissue and its blood vessels, which gives an indication of the forces to which these structures were exposed.

It was found that tensile, compressive and torsional forces invariably produce a pathological tensile stress field within the cord parenchyma.

Our knowledge of the existence of these stress fields is now sufficient to afford a guide to the appropriate surgical measures by means of which the stress acting within the cord parenchyma can be counteracted or eliminated, thereby promoting the blood circulation and improving neural conductivity. It would seem evident that it is the deformation within the conducting axon cylinders that is the prime cause of reversible neurological symptoms.

An outline of our present knowledge of the biomechanical behaviour of the cord is now given to illustrate my thesis. It is the movement of the cord that in the presence of pathological processes are ultimately responsible for neurological symptoms. The pons, medulla oblongata and the cord, with their cranial and spinal nerve roots, may be regarded as a continuous tissue tract, which is firmly anchored rostrally at the mesencephalon and caudally by the attachments of the nerve roots. As a result of this anchorage, the tract adapts itself immediately and 
continuously to the shortening and elongation of the spinal canal during dorsiflexion and ventriflexion respectively. This adaptation occurs through internal changes in the shape of the component structures of the parenchyma. Regarding the many structures involved in this process of adaptation, reference will only be made to the fact that the collagenous elements are arranged in a rhomboid structure, whose angles of intersection change during elongation of the canal and hence enable the cord to lengthen. When the head and spinal column are in the erect position, all the elastic components that have become folded and have thus formed a 'tissue reserve' during dorsiflexion, are pulled out smoothly. When the head and column are ventriflexed the elastic components are further extended, and their elasticity is now exploited. In Figure $\mathrm{I}$, the folding of the axis cylinders is seen as

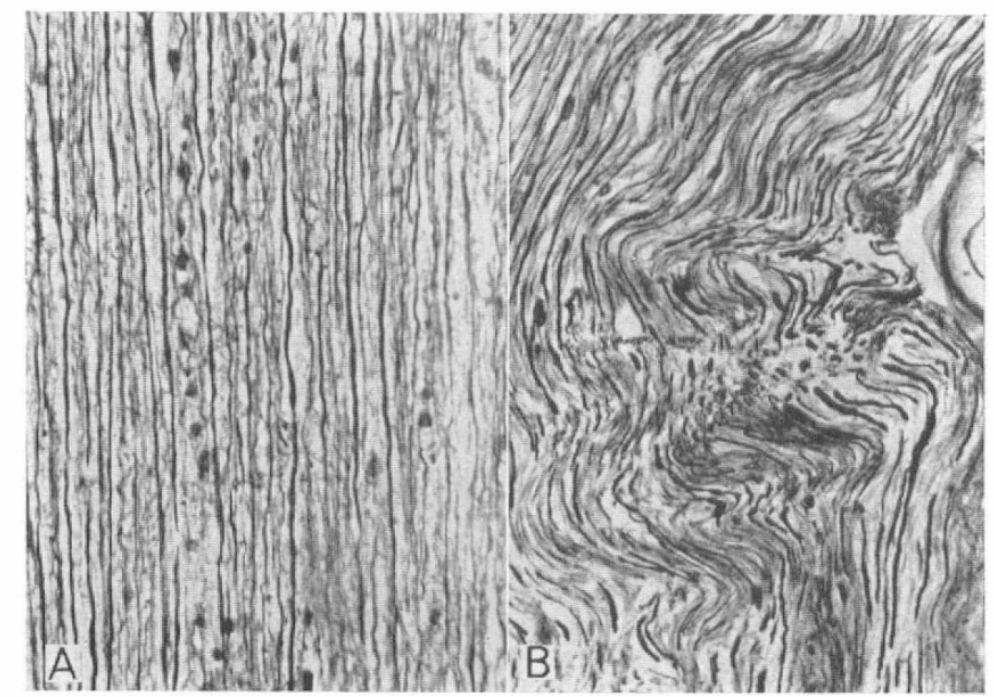

FIG. I

Sagittal section through the cervical spinal cord at the level of C6. Dorsal aspect. Palmgren's stain. $\times 370$. A, On ventriflexion of the cervical spine the parenchymal network is drawn out and the axis cylinders straighten. B, On dorsiflexion the axis cylinders fold threedimensionally (Breig, I960).

the tract comprising the pons and cord is shortened (fig. I, A), and their extension as the tract lengthens again (fig. $I, B$ ).

An observation of major practical significance is the slackening of the ponsspinal cord with their nerve roots, as the spinal canal is shortened. The fact that the slackness is not evident to the naked eye would account for it having been overlooked for so long. From the neurosurgical literature it is evident that the opposite process, namely the stretching of the cervical cord when the head and cervical column are flexed forwards, has long been recognised; in fact, many authors have complained that this is a source of difficulty in operations on the ventral aspect of the cervical canal, and incurs a risk of damage to the cord. As we now know today, this can easily be avoided altogether by simply dorsiflexing the cervical spine, so that both the cervical cord and its nerve roots are slackened. In this state the cord 
can be raised from the canal and drawn to the side to the full extent permitted by the nerve roots.

Although the author described the slackening phenomenon as long ago as I957, it was only in 1960 that he required to test it in practice in an operation for a clivus meningioma. In 1956, Dodge in the Mayo Clinic wrote that 'although most tumours in the foramen magnum region are benign, any attempt to remove them may result in no more than a Pyrrhic victory'. In our case the meningioma was an extremely large one, as was seen in the air myelogram. The tumour forced the pons and the medulla oblongata backwards in a large arc. It appeared obvious to us that this displacement set up powerful tensions in the pons and medulla oblongata in both axial and transverse directions. After removing the bone and uncovering the dura we dorsiflexed the head and neck, a manipulation that now is greatly facilitated by a specially designed head support. Apart from the fact that the space available for surgery became more restricted, nothing in particular could be seen with the naked eye, but on insertion of brain spatulae it appeared to be much easier to move the pons and medulla oblongata to the side. The tumour could then be removed by piecemeal extirpation without difficulty. After the operation, spontaneous respiration was resumed, though it had been expected that respirator treatment might be necessary. After about 14 days the patient had almost fully recovered from her tetraspasticity, defective sensibility and urinary incontinence. During the past decade we have used this spinal cord relaxation technique with advantage to both patient and surgeon in operations at sites located from the posterior cranial fossa to the sacral end of the spinal canal.

A long-standing impediment to the recognition of the nature of the forces acting on both the spinal cord and the nerve roots in the individual situation has been the view, still adhered to today, that all mechanical stresses on the spinal cord and nerve roots is compressive. This myth was finally exploded in 1963 by Marions and the author in the examination of 120 patients with a suspected intervertebral disc herniation, by means of a water-soluble contrast medium which was injected into the lumbosacral canal. To ascertain what actually happens to the spinal cord and its nerve roots as a consequence of the forces set up by the herniation, the conventional radiographs were supplemented by two extra radiographs of the lumbar region, with the cervical spine firstly in dorsiflexion and then in ventriflexion (fig. 2). It was then clearly seen that during dorsiflexion the medullary cone fell some millimetres in the caudal direction owing to the relaxation of the spinal cord and the simultaneous slackening of the nerve roots, $(a)$, while on ventriflexion of the head and the cervical spine the cone and the nerve roots were straightened $(b)$. In disc herniation the nerve root is pulled against the disc protrusion and at the same time stretched over it; and what is just as important, within the spinal canal in these studies there was no evidence of any osseous or other structure opposite the protrusion that could have involved the nerve root in a 'pincer action'. This prompted us to carry out our first examination of the type of mechanical stress that is exerted by a disc protrusion on the parenchyma of a nerve root or of the spinal cord under the conditions just outlined. The macroscopic changes and effects will be considered first. During the phase when the spinal canal is undergoing elongation, any ventrally or anterolaterally located protrusion exerts a pressure on the nearest section of the pons-spinal cord-nerve root structures: this pressureisgreatest in the region or plane of contact and diminishes to zero on the opposite free surface of the section of the structures in question. At the same time the protrusion exerts 
a tension on the section of the neural structures that are curved and stretched over it. This tension is least along the aspect in contact with the protrusion and greatest along its free aspect. From an analysis of a large number of pathological changes that exert a unilateral pressure on some part of the stretched pons-spinal cord

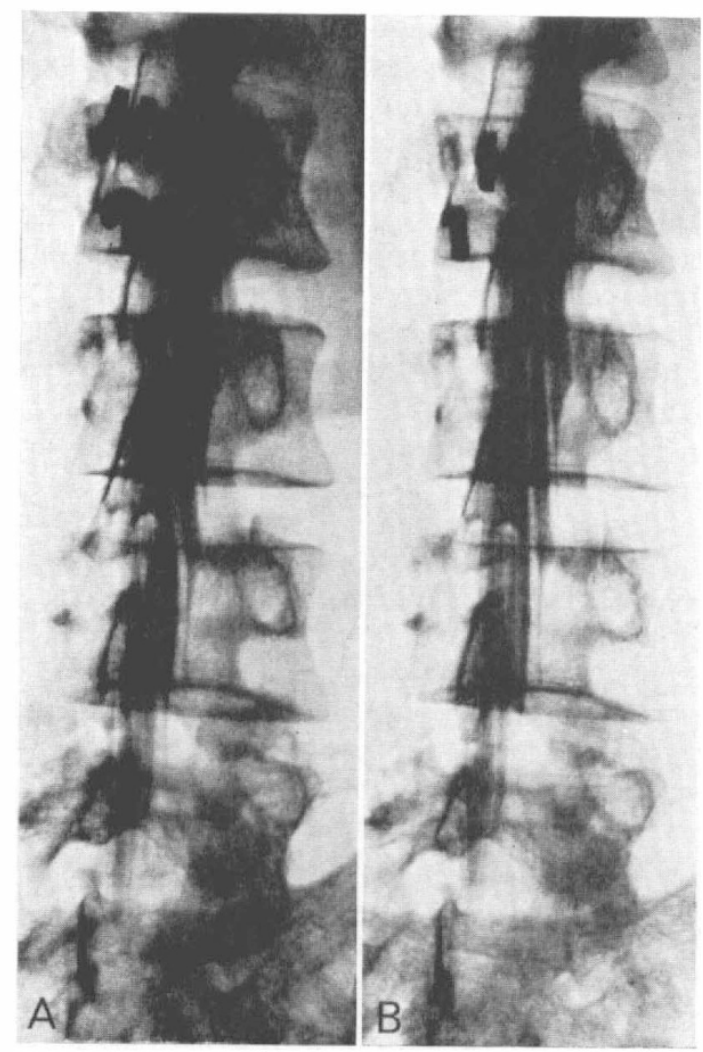

FIG. 2

The propagation of the pons-spinal cord tissue tract's slackening and stretching to the lumbosacral nerve roots visualised by water-soluble contrast injection into the lumbar subarachnoidal space. A, In dorsiflexion of the cervical spine the nerve roots form a smaller angle with the margins of the vertebral bodies than in ventriflexion (B). The nerve roots are respectively slackened and stretched.

system it would appear that the stress can invariably be reduced to what might be described as the thrust effect of an impinging body.

Histological sections through an arbitrary area of the pons and spinal cord show that when they are stretched all unyielding pathological changes that are located outside, or more accurately ventral to, or within its parenchyma (from the region of the incisura tentorii to the sacral canal), give rise to the same phenomenon, namely pressure on and vaulted narrowing of the extended nerve tissue. As a 
result of this thrust the strata running parallel to the transverse or sagittal sectors of the impinging body move closer to one another, while their tendency to crowd together decreases with the distance from the impinging body. The effect of an inclusion body has been studied by inserting a small metal cone in the cord tissue
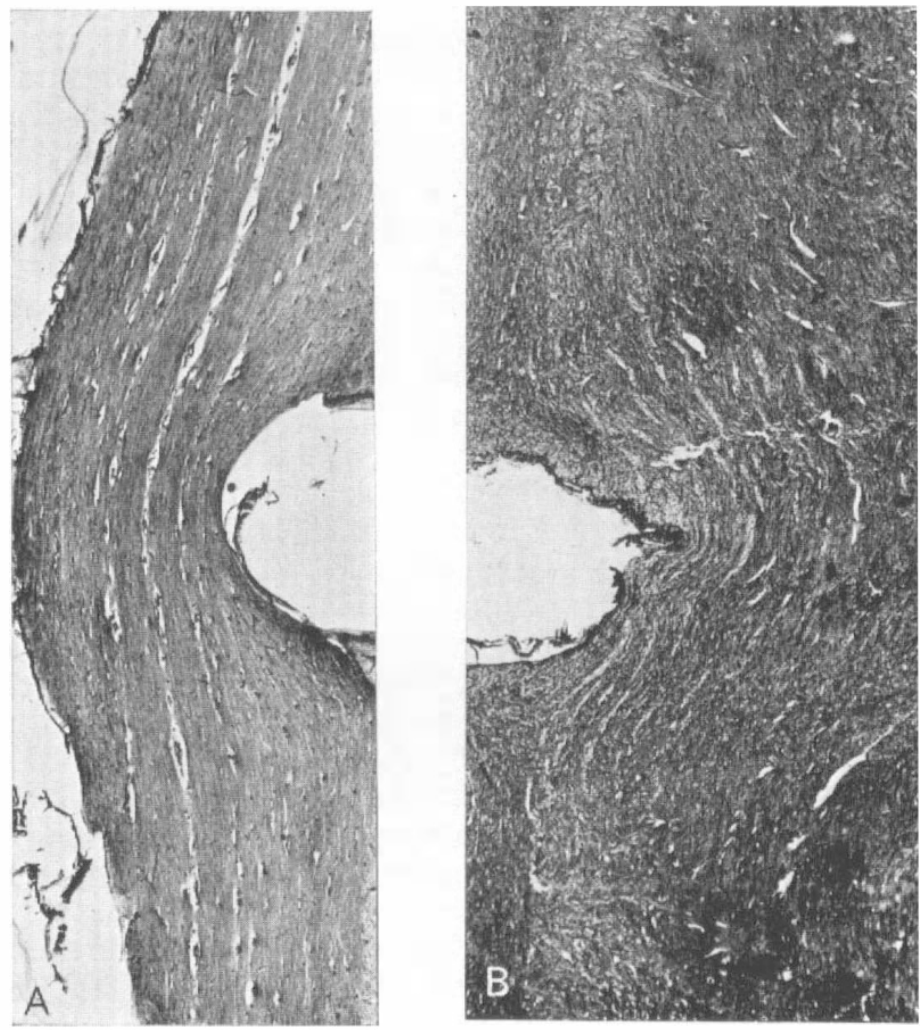

FIG. 3

Tensile stress field within the cervical spinal cord artificially produced by inserting a small metal cone into the cord tissue and fixing it in situ by injection of formalin into the subarachnoidal space. Section in front of the coronal mid-plane. Numerous branches of the central arteries with their surrounding VirchowRobin's space are met. A, On ventriflexion of the cervical spine the fibres bend and stretch over the cone. $\times 19^{\circ} 4$. B, On dorsiflexion of the cervical spine (in another case) the fibres slacken and leave their contact with the cone. The tensile stress field is then dissolved. $\times$ I0'0.

in situ (fig. 3). Histological studies show that it is possible to follow the whole of a typical tensile stress field set up on ventriflexion of the spinal column. The field is indicated by the decreasing extent to which the fibres are packed and by the reduction in their bending with the distance from the impinging body towards the periphery. In the present ideal case (so far as the visible manifestations of the histodynamic stress are concerned) the direction of the tissue fibres corresponds to the lines of force of the stress field. All the evidence indicates that it is the tension 
in, and bending of, the conducting nerve elements with the accompanying decrease in their cross-section and the corresponding increase in their resistance to conduction that account for the neurological symptoms. When the spinal canal is elongated the deformation of the parenchyma usually increases directly as the tension in the cord, and as we know symptoms can sometimes derive from adjacent pathways. As has already been stated above, the neurological symptoms may often disappear; that is to say, when the fibres slacken and the tensile and bending strain has been relieved their conductivity can recover. The return of conductivity of the ulnar nerve that may be achieved by its relaxation by operative transposition demonstrates a familiar parallel in the peripheral nervous system.

It is, however, not only the strain in the actual conducting nerve elements that accounts for the neurological symptoms, but also the hypoxia of the nerve cells that occurs when the lumen of their supplying blood vessels (as integral components of the parenchyma) are reduced by being stretched, if they happen to run parallel to the axis of the tension strain field. A typical example where the symptoms of neurological deficit are evoked by a critical reduction of the lumen of blood vessels is found in the atrophy of nerve cells of the lateral pyramidal tracts, a process that sometimes occurs in cervical spondylotic myelopathy. These cells are, of course, located quite a distance from the osteophytes on the ventral aspect of the cervical canal that are responsible for the stress in question. In a study that Turnbull and the author made in 1966 to analyse this situation, it was found that the branches of the central arteries that supply the pyramidal tracts happen to run parallel to the axis of a tension strain field oriented in the transverse direction of the cervical cord. If the tension in the parenchyma is great enough and the resulting narrowing of the blood vessels has led to protracted hypoxia within the corresponding region, the hypoxia can ultimately lead to atrophic changes of the lateral pyramidal tracts. This would seem to afford a plausible explanation of the impairment of an area of parenchyma remote from the osteophytes that are responsible for the pathodynamic strain.

The tensile stress field mentioned earlier in this paper can be resolved by the relaxation of the pons-spinal cord complex, as is evident from, for instance, the rapid regression of the neurological symptoms following removal of a clivus meningioma. In the case of smaller lesions the relaxation of the tract required for relief of the tensile and bending stress can be procured by simply dorsiflexing the cervical spine.

The tension in the nerve fibres produced by the unilateral thrust of an impinging body acting on the parenchyma of a stretched spinal cord either from without or from within towards the pia is usually of moderate degree. In the case shown in Figure 4, the tensile stress has been exerted by an osteophyte located ventrally in the cervical canal. In the histological section through the coronal plane of the cervical spinal cord in the specimen (see fig. 3), the thrust of the nerve fibres exerted by an intraparenchymally located unyielding body has been reproduced by inserting a small metal cone in the cord tissue. The effect of this cone and that of the osteophytes on the parenchyma during physiological stretching of the spinal cord, when the spine was flexed forwards is shown (fig. 4); (tissues fixed by subarachnoidal formalin injection in situ).

In contrast to a thrust's moderate tensile and bending stress that can usually be resolved by slackening the spinal cord, bilateral constriction of nerval tissue through compression, or more obviously, a pincer action always produces an 
extremely high axial tension (the resultants of which are directed in cranial and caudal directions) in a limited section of the tissue. Under certain conditions, this tension will inevitably result in rupture of the cord substance. This has not hitherto been fully explained.

The mechanical effect of a pincer action on an elastic body may be examined by means of a stress optical model. As is indicated in the isochromes in a photograph,

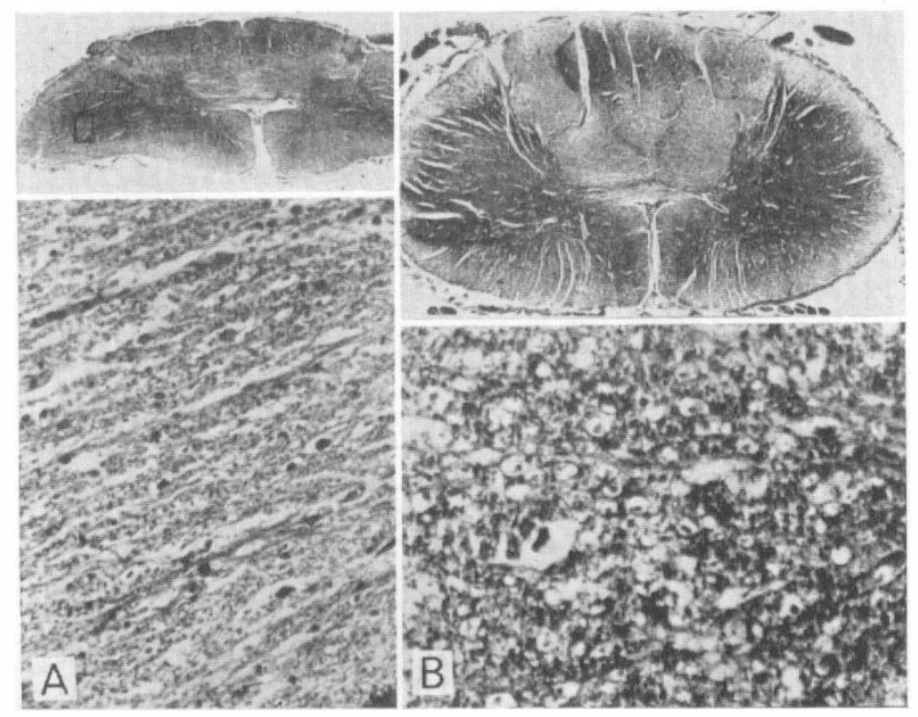

FIG. 4

A, (upper); Transverse section through a tensile stress field in the right side of the cervical spinal cord (left side disregarded) produced by the unilateral thrust of the right part of a dumbbell shaped osteophyte located ventral to the cord. Lower: magnified (framed) part of the tensile stress field from above. $\times 80$. $\mathrm{B}$, (upper and lower); Transverse section through cervical spinal cord parenchyma at the identical segmental level as in specimen (A), but formalin fixated in the erect position. Normal histologic picture without evidence of any mechanical stress. Lower left and right specimen; Whilst in the unloaded state the parenchyma has a honeycomb-like appearance, the laterad-towards the dentate ligament-directed tension in the left specimen has led to a clear stratification. In spite of the fact that the layers are more packed in the left specimen, there appears a clearly visible intercellular space and due to the transverse widening a bigger interspace can be discerned between cell-bodies and transversally cut axis cylinders.

taken of the compressed body through a polarising glass plate, an axial tension is set up in a short segment of the body (the information provided by the isochromes concerning differences in the material can be transposed into a graph).

To examine the effect on the parenchyma of a pincer action on the spinal cord the following experiment has been performed. By means of the registration technique mentioned above in a few of the $2 \mathrm{I}$ post-mortem specimens with the cervical spine in the dorsiflexed position, the cervical cord was compressed to exert 
a pincer action. This was achieved by pushing a round wooden plug towards the anterior aspect of the dura and cord through a hole drilled between the $C_{5}$ and C6 vertebrae; in others of these specimens a similar pincer action on the cervical

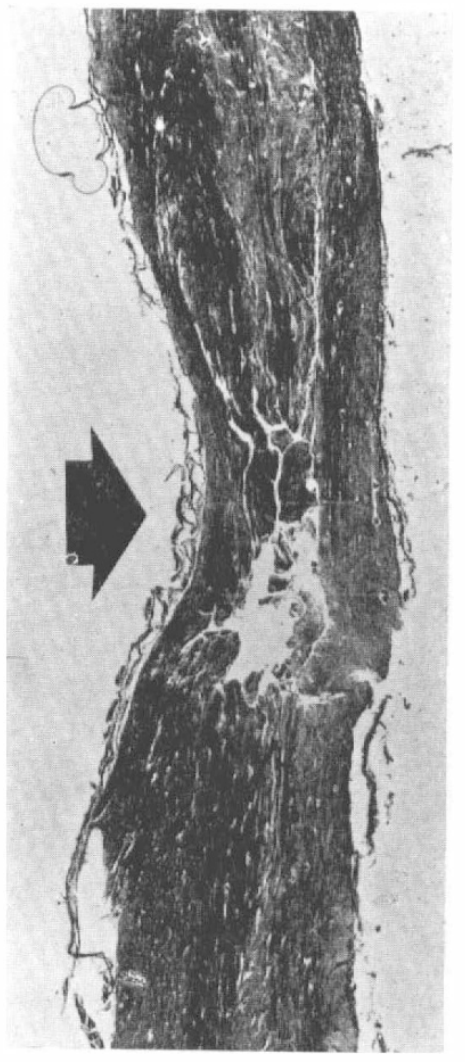

FIG. 5

Tearing of the dorsal aspect of a cervical spinal cord specimen produced in situ by a wooden plug moving in the anteroposterior direction with the cervical spine kept in maximum dorsiflexion. Only one-eighth of the width of the parenchyma at the ventral aspect of the cervical spinal cord still consists of coherent (undamaged) fibres. This type of damage has hitherto been ascribed to a protruding flaval ligament. cord was exerted by inserting the plug from behind. Pressure great enough to reduce the diameter of the cervical cord by about 20 per cent. usually tore the parenchyma, irrespective of whether the compression had been exerted extremely slowly or as rapidly as the method permitted.

When the plug was pushed in from behind towards the anterior wall of the cervical canal tearing of the ventral aspect of the cervical cord always occurred. This contradicts the view held hitherto that in the so called hyperextension injury it is the flaval ligaments that are responsible for the tearing of the dorsal aspect of the cervical spinal cord. In fact, this must be due to an unyielding body moving in the anteroposterior direction and consisting either of an intervertebral disc or of an increased volume of cerebrospinal fluid (fig. 5). The unyielding body can be reliably identified only by means of rapid cinematography.

The material in which it has been possible to obtain clinical experience of the special therapeutic possibilities offered by slackening of the spinal cord is limited to 4 patients; 3 of them suffered from cervical spondylotic myelopathy and I from the sequelae of a central spinal cord injury. This latter patient has been operated upon too recently to warrant inclusion in the survey of the results.

In 2 of these patients within a few days after surgery tetraspasticity diminished markedly and sensibility and bladder continence returned. In the other 2, central hemiparesis improved, and there was better control of bowel function.

Regarding the occurrence of a complete interruption of conductivity of certain spinal cord pathways the myelopathy cases do not meet the criteria that one usually places on the term 'incomplete cord lesions'. But the neurological symptoms in these cases are due to histodynamic stress similar in principle to that which would be expected in incomplete or seemingly complete transverse lesions. For recovery of the conductivity of certain neural pathways due both to spinal cord injury and cervical myelopathy the nerve fibres must not, of course, be torn. It is thus the preserved anatomical continuity of some or all of the nerve fibres and the increased tension in these fibres with subsequent impairment of 
conductivity that are the common factors in these heterogeneous cord affections. If the respective nerve fibres in these two types of lesion are neither torn nor atrophied but their conductivity is still reduced or abolished completely, there will probably be a histodynamic strain similar to that produced by a unilateral thrust. On the basis of these experimental and clinical observations it would appear to be justified today to infer that under these particular conditions of histodynamic strain that there is a theoretical chance that recovery of the conductivity of the neural parenchyma can be secured by relaxation.

An outline of the essentials of the surgical technique for relaxing the pons and spinal cord will now be given. Gas myelography is carried out prior to operation to rule out or demonstrate the presence of pathological formation within the spinal
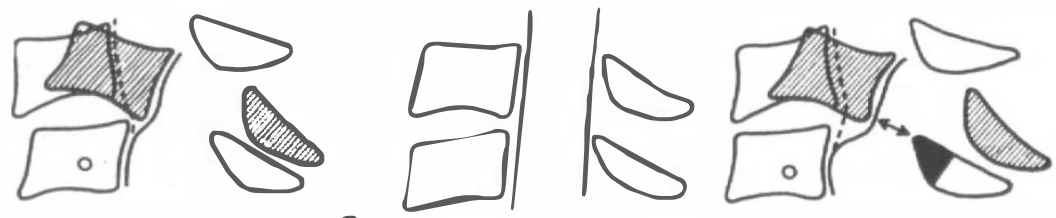

a

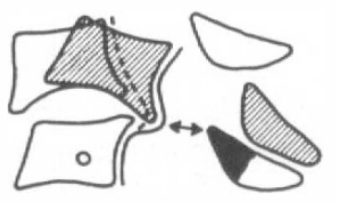

C

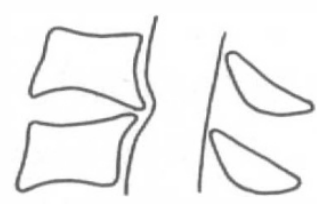

FIG. 6

Schematic representation of the amount of resection of the upper rim of the vertebral arch (arco-cristectomy) necessary for alleviation of its pincer action on the spinal cord in the presence of another encroaching protrusion (osteophyte) in the ventral wall of the canal. The part of the vertebral arch shaded in the diagrams must be incised and then lifted up from the dura. No instrument is to be inserted between the bone and the dura mater as any further encroachment on the nerval elements immediately increases the tensile stress in the parenchyma with irreversible damage as the inevitable consequence.

canal that may be compressing the cord or nerve roots or that may do so on dorsiflexion of the cervical column; or to show cervical spinal canal stenosis. If such formations are found, selective resection by a special conservative method (arcocristectomy) must be performed at the appropriate level. This operation can be limited to removing the body, even just the upper border of a vertebral arch, which is responsible for a pincer action (fig. 6). In accordance with the view just expressed regarding the histodynamic tension it is felt that it may be eliminated simply by a transplant of fascia lata and anchoring it between the external occipital protuberance and the spinous process of the TI. For the sake of histocompatibility autografts have been used exclusively. In 2 of the patients available for follow-up examination, the slight dorsiflexion of the cervical column was retained for more than 3 years by means of a fascial graft. It would thus seem that this method is also mechanically successful. If we accept the reduction or the disappearance of neurological symptoms such as spasticity, impaired sensibility and urinary 
incontinence as criteria of the therapeutic result, then those obtained with this method so far may be classed as 'satisfactory' to 'extremely good'.

With a special instrument the degree of dorsiflexion of the cervical spine required to obtain a slack cord can be determined. This was demonstrated in I of the 3 patients with cervical myelopathy operated on in April 1970. He had had a spastic hemiparesis and a corresponding spastic gait, impaired sensibility below the iliac crests and slight urinary incontinence. A few days after the operation these manifestations had appreciably diminished, and after a fortnight they had disappeared almost completely. The patient is very satisfied with the result.

When the patient was trying to bend his cervical spine forwards as far as possible, the head could be brought into the erect posture, which is slightly forward of the intended position.

This surgical method constitutes a new active conservative measure for the treatment of spinal cord disorders, both those caused by extramedullary unyielding processes in the spinal canal, and intraparenchymal changes including the scarring resulting from spinal cord trauma. This surgical approach is fully consistent with the conservative principles adopted by Sir Ludwig Guttmann. But I venture to suggest that it is more direct, and more far reaching and radical, since it effectively eliminates any harmful tensile stress in the spinal cord parenchyma that must inevitably arise from the lengthening of the cervical spinal canal that occurs when the neck is flexed forwards.

\section{SUMMARY}

The author is firmly convinced that by relaxing the pons and the spinal cord a number of disorders of the central nervous system are rendered accessible for symptomatic treatment, amongst them, intraparenchymal affections. Just which of them, and how many cases of, for instance, multiple sclerosis and sequelae of spinal cord injury can benefit from this method can only be established by practical experience. The accumulation of such experience is a challenging task.

A number of misconceptions have hitherto stood in the way of a realistic assessment of the tensile forces to which spinal cord tissue is submitted during functional movements of the spine in the presence of unyielding pathological changes. An insight into what happens in reality opens up a new practical approach to treatment, namely relaxation of the spinal cord by a fascia lata graft and where necessary, elimination of the pincer action by resection of the offending structure.

\section{REFERENCES}

BREIG, A. (1960). Biomechanics of the Central Nervous System. Stockholm, Sweden: Almqvist \& Wiksell.

BREIG, A. (1970). F. Biomech. 3, 7-9.

BREIG, A. \& MARIONS, O. (I963). Acta rad., 1, 6, I I4I-I I60.

Breig, A., Turnbull, I. \& Hassler, O. (I966). F. Neurosurg. 25, I, 45-56.

DODGE, I. W. I. (1956). F. Neurosurg. 13, 603. 